Check for updates

Cite this: J. Mater. Chem. A, 2017, 5 , 25249

Received 26th August 2017 Accepted 13th November 2017

DOI: $10.1039 / \mathrm{c} 7 \mathrm{ta0} 528 \mathrm{f}$

rsc.li/materials-a

\section{Remarkably simple achievement of superhydrophobicity, superhydrophilicity, underwater superoleophobicity, underwater superoleophilicity, underwater superaerophobicity, and underwater superaerophilicity on femtosecond laser ablated PDMS surfaces $\dagger$}

\author{
Jiale Yong, (D) a Feng Chen, (DD *a Minjing Li, ${ }^{\mathrm{b}}$ Qing Yang, ${ }^{\text {tb }}$ Yao Fang, ${ }^{\mathrm{a}}$ Jinglan Huo ${ }^{\mathrm{a}}$ \\ and Xun $\mathrm{Hou}^{\mathrm{a}}$
}

A simple achievement of six different super-wettabilities on the femtosecond laser ablated polydimethylsiloxane (PDMS) surface is reported for the first time. Both the experimental and theoretical analysis revealed that underwater oil wettability and underwater bubbles' behavior on a solid surface are closely related to the in-air water wettability of the substrate surface. The original femtosecond laserinduced microstructured PDMS surface exhibited excellent superhydrophobicity in air and generally became superoleophilic and superaerophilic in water. After being further irradiated by oxygen plasma, the rough PDMS surface switched to superhydrophilic. Underwater superoleophobicity and superaerophobicity could be exhibited when such a superhydrophilic PDMS surface was immersed in water. Furthermore, various superhydrophobic-superhydrophilic, underwater superoleophobic-superoleophilic and underwater superaerophobic-superaerophilic hybrid patterns were successfully designed and achieved on the femtosecond laser ablated PDMS surface by subsequent selective oxygen plasma treatment. We believe that the reported preparation principle of superhydrophobic, superhydrophilic, underwater superoleophobic, underwater superoleophilic, underwater superaerophobic, and underwater superaerophilic surfaces would have important guiding significance to researchers and engineers to effectively control water droplets, oil droplets and the behavior of bubbles on a material surface.

\section{Introduction}

Solid, liquid, and gas are three basic states of matter and they usually interlace with one another to form different kinds of solid/liquid/gas interfaces. Super-wettability at the solid/liquid/ gas interface has great theoretical and practical significance. ${ }^{1-7}$ The materials with special wettability have a wide range of applications, including liquid repellence, ${ }^{2-4}$ self-cleaning coating, ${ }^{8,9}$ oil/water separation, ${ }^{10-13}$ anti-corrosion, ${ }^{14}$ antifogging/icing, ${ }^{15-17}$ water/fog harvest, ${ }^{18}$ microdroplets manipulation, ${ }^{19-22}$ lab-on-a-chip, ${ }^{23,24}$ microfluidic, ${ }^{25,26} \mathrm{drag}$ reduction, ${ }^{27}$ and cell engineering..$^{28,29}$ The most widely-studied superwettability is

${ }^{a}$ State Key Laboratory for Manufacturing System Engineering, Key Laboratory of Photonics Technology for Information of Shaanxi Province, School of Electronics \& Information Engineering, Xi'an Jiaotong University, Xi'an, 710049, PR China. E-mail: chenfeng@mail.xjtu.edu.cn

${ }^{b}$ School of Mechanical Engineering, Xi'an Jiaotong University, Xi'an, 710049, PR China.E-mail: yangqing@mail.xjtu.edu.cn

$\dagger$ Electronic supplementary information (ESI) available. See DOI: $10.1039 / \mathrm{c} 7 \mathrm{ta} 07528 \mathrm{f}$ superhydrophobicity..$^{30-32}$ A superhydrophobic surface such as a lotus leaf has excellent water repellence and self-cleaning ability. ${ }^{33,34} \mathrm{~A}$ water droplet on the superhydrophobic surface can maintain a spherical shape and easily roll away. Since the underwater superoleophobicity of fish scales was discovered, the study related to underwater oil wettability has been attracting increasing attention. ${ }^{1,35,36}$ The underwater superoleophobic materials are able to repel oils in water medium. The opposites of superhydrophobicity and underwater superoleophobicity are superhydrophilicity and underwater superoleophilicity, respectively. As another important case of the underwater gas/liquid/ solid interface, gas can appear in water in the form of bubbles. Controlling the bubbles' behavior on a material surface also has important application prospects. ${ }^{37-39}$ Two extreme cases (underwater superaerophobic surface and underwater superaerophilic surface) are extraordinary owing to their anti-bubble or bubbleabsorption property. Superhydrophobicity, superoleophobicity or superaerophobicity refers to a small water droplet, oil droplet, or bubble on a substrate surface with a contact angle (CA) larger than $150^{\circ}$, respectively. ${ }^{2-4}$ Correspondingly, superhydrophilicity, 
superoleophilicity, or superaerophilicity can be defined as exhibiting CA less than $10^{\circ}$ with a water droplet, oil droplet, or bubble, respectively. Although one or two aspects of the abovementioned six super-wettabilities can be realized on one sample surface by typical microfabrication technologies, ${ }^{\mathbf{1 - 4}}$ a simple method that can simultaneously achieve superhydrophobicity, superhydrophilicity, superoleophobicity, superoleophilicity, superaerophobicity, and superaerophilicity on the same sample has not been reported until now.

In this paper, polydimethylsiloxane (PDMS) surface was ablated by femtosecond laser to form a hierarchical rough microstructure. The structured PDMS surface exhibited excellent superhydrophobicity in air. When the sample was immersed in water, the underwater superoleophilicity and superaerophilicity was exhibited. If such rough PDMS surface was further irradiated by oxygen plasma for a short time, the wettability of the sample would change significantly. Herein, the femtosecond laser-induced rough PDMS surface exhibited superhydrophilicity in air, and superoleophobicity and superaerophobicity in a water medium. Therefore, six different superwettabilities can be easily achieved on such laser structured PDMS surface. Based on the wettability transition after oxygen plasma treatment, a new strategy for constructuring wettabilitycontrast domains on the same femtosecond laser ablated PDMS surface was proposed by using selective oxygen plasma treatment.

\section{Experimental section}

\subsection{Femtosecond laser ablation}

The PDMS thin films were prepared by mixing the pre-polymer and curing agent (Dow Corning Corporation) (v/v = $10: 1$ ), followed by curing at $100{ }^{\circ} \mathrm{C}$ for $2 \mathrm{~h}$. Femtosecond laser ablation was used to generate microstructures on the PDMS surface. Fig. 1 shows the setup of femtosecond laser microfabrication system. The PDMS sample was fixed on a computer controlled three-dimensional (3D) processing platform in advance. The laser beam (center wavelength $=800 \mathrm{~nm}$, duration $=50 \mathrm{fs}$, and repetition rate $=1 \mathrm{kHz}$ ) originating from a Ti:sapphire laser system (Libra-usp-he, Coherent, America) was focused on the PDMS surface by an objective lens with NA of 0.40 . In the experiment, the typical line-by-line (serial) scanning manner was adopted as shown in the lower right inset of Fig. 1. The laser power used was $40 \mathrm{~mW}$. The sample was ablated by the femtosecond laser beam at the scanning speed of $5 \mathrm{~mm} \mathrm{~s}^{-1}$ and the shift of scanning lines of $5 \mu \mathrm{m}$. Following this, the sample was cleaned with acetone, alcohol and deionized water via ultrasonication.

\subsection{Oxygen plasma treatment}

The femtosecond laser structured PDMS surface was irradiated by oxygen plasma in a PR-3 plasma reactor (Heshi Technology, China) for $30 \mathrm{~s}(50 \mathrm{~W})$ to activate the PDMS surface and switch the surface from superhydrophobic to superhydrophilic.

\subsection{Characterization}

The microstructure of the PDMS surface was observed by a scanning electron microscope (Gemini SEM 500, Zeiee, Germany). The water wettability, underwater oil wettability, and underwater behavior of bubbles on the femtosecond-laserinduced hierarchical rough PDMS surface were systematically investigated using a contact-angle system (JC2000D, Powereach, China). The typical volumes of the tested water droplet, oil droplet, and bubble are $\sim 8 \mu \mathrm{L}, \sim 8 \mu \mathrm{L}$, and $\sim 3 \mu \mathrm{L}$, respectively. The dynamic processes of absorbing a water droplet, oil droplet or a bubble were captured by a high-speed CMOS camera (CAMMC1362, Mikrotron, Germany) with the maximal frame rate of $2000 \mathrm{fps}$.

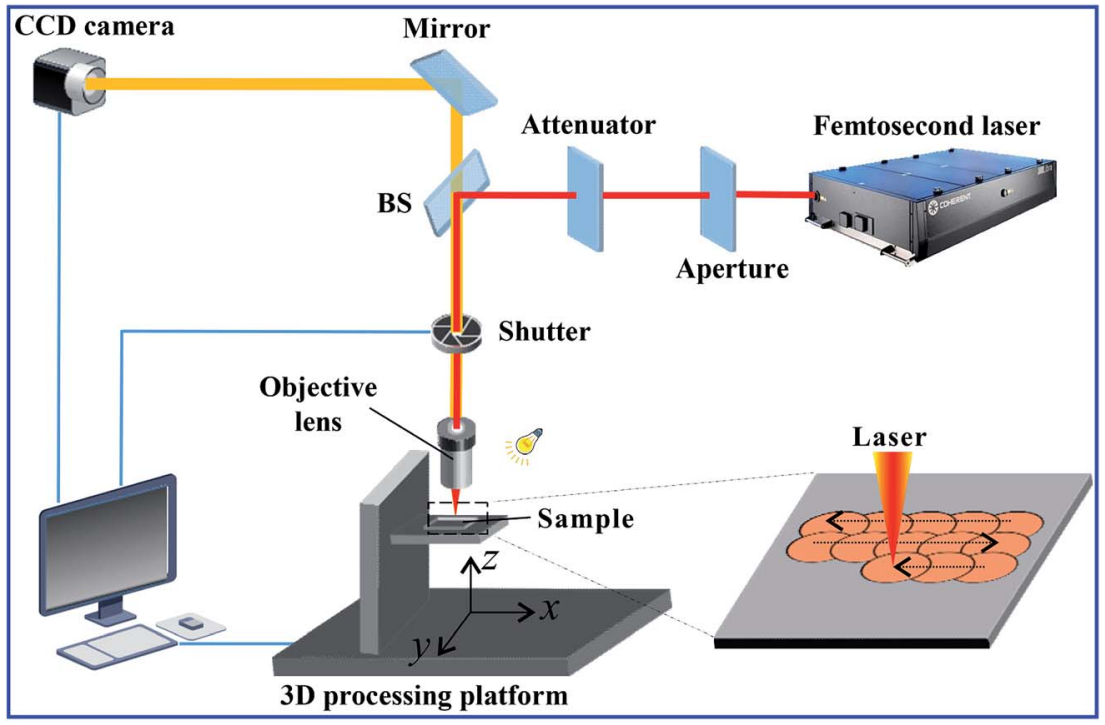

Fig. 1 Schematic diagram of the experimental setup. 


\section{Results and discussion}

Femtosecond laser microfabrication is emerging as an effective technology to form two- or three-dimensional microstructures on material surfaces. ${ }^{7,40-42}$ This technology has numerous advantages, such as extensive material processing, high precision processing, and strong controllability. ${ }^{7,40-42}$ Since the surface wettability is primarily governed by the surface microstructures and chemical composition, recently, femtosecond laser microfabrication has also been applied to control the surface wettability of solid materials because different micro/ nanoscale hierarchical structures can be directly created on various material surfaces by a simple one-step femtosecond laser scanning process. ${ }^{743-48}$ Fig. 2 shows the scanning electronic microscopy (SEM) images of the PDMS surface after femtosecond laser ablation. There are a numerous coral-like microstructures with the size of several micrometers distributed on the PDMS surface (Fig. 2a). The surface of the microscale corals is further decorated with abundant nanoscale protrusions (Fig. 2b), forming a micro/nanoscale hierarchical rough structure.

The water wettability, underwater oil wettability, and underwater bubble's behavior on the femtosecond-laserinduced hierarchical rough PDMS surface were systematically investigated. It has been previously reported by our group that
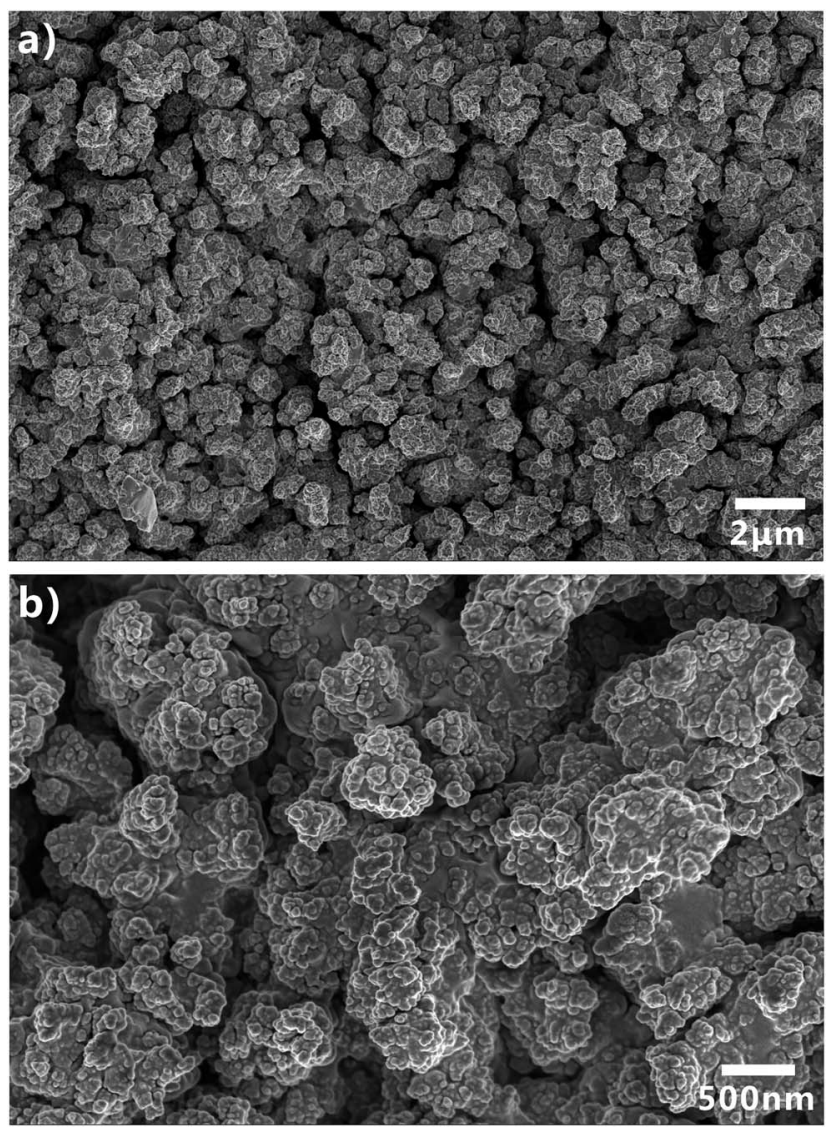

Fig. 2 SEM images of the PDMS surface after femtosecond laser ablation. femtosecond laser structured PDMS surfaces display excellent superhydrophobicity in air. ${ }^{20,49,50}$ As shown in Fig. 3a, a small water droplet that was placed on the rough PDMS surface could remain spherically shaped all the time. The CA value to the water droplet is $155.5^{\circ} \pm 1.5^{\circ}$. The droplet would easily roll away as long as the substrate was tilted by $2^{\circ}$, revealing a very low adhesion between the water droplet and the femtosecond laser ablated PDMS surface (Fig. 4a and Movie S1 in ESI†). When such superhydrophobic PDMS sample was immersed in water, a silver mirror-like reflectance on the laser ablated area could be visually observed (Fig. 3g). This mirror-like interface is ascribed to an air layer trapped between water and the rough microstructures of the PDMS surface. ${ }^{51,52}$ This phenomenon is another way of demonstrating that water droplet on such rough PDMS surface is at the Cassie wetting state, independent of simultaneously exhibiting superhydrophobicity and ultralow water adhesion. ${ }^{1,53}$ PDMS is an intrinsically hydrophobic

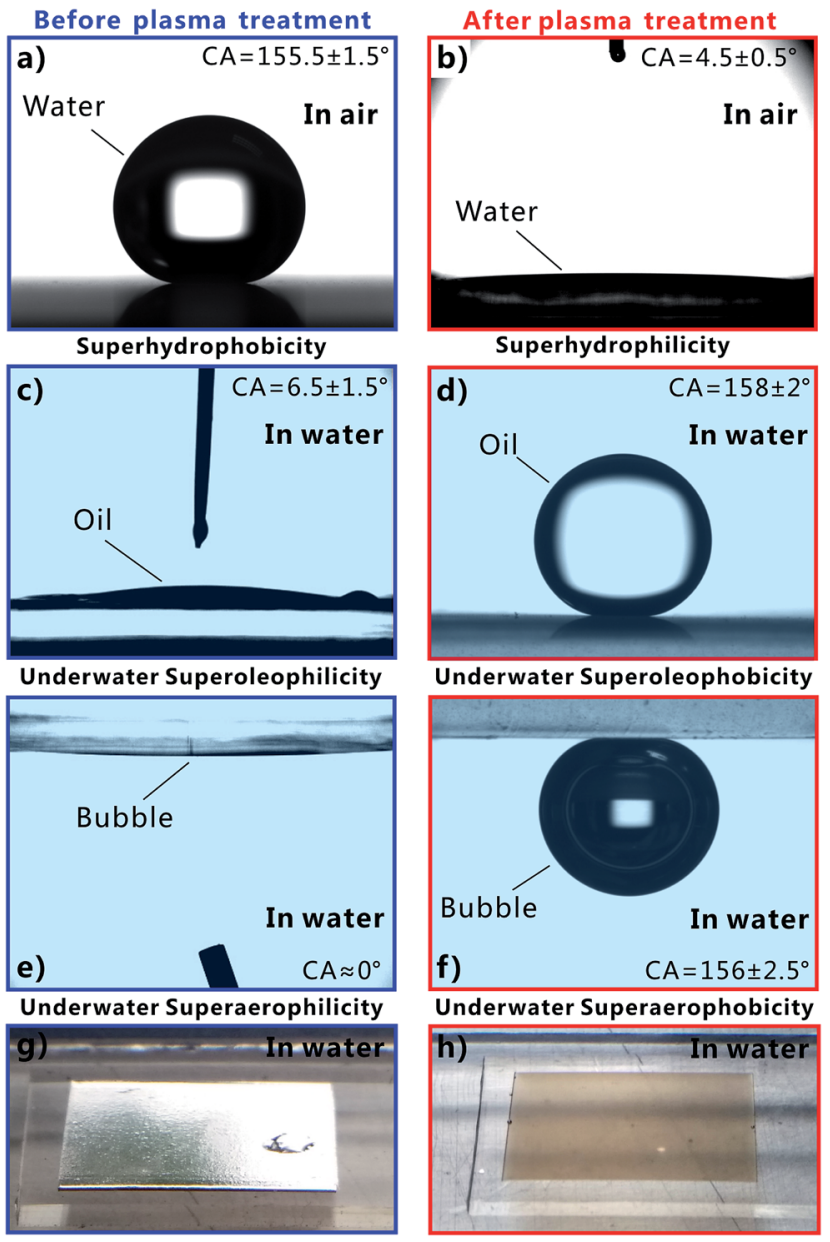

Fig. 3 Static water wettability, underwater oil wettability, and underwater bubble's behavior on the femtosecond-laser-induced hierarchical rough PDMS surface. $(a, b)$ Water droplet on the sample surface in air. (c, d) Oil droplet on the sample surface in water. (e, f) Bubble on the sample surface in water. $(\mathrm{g}, \mathrm{h})$ Photograph of the sample surface in water. $(a, c, e, g)$ are for the PDMS surface that was just ablated by femtosecond laser. (b, $d, f, h)$ are for the PDMS surface that was ablated by femtosecond laser and further irradiated by oxygen plasma. 


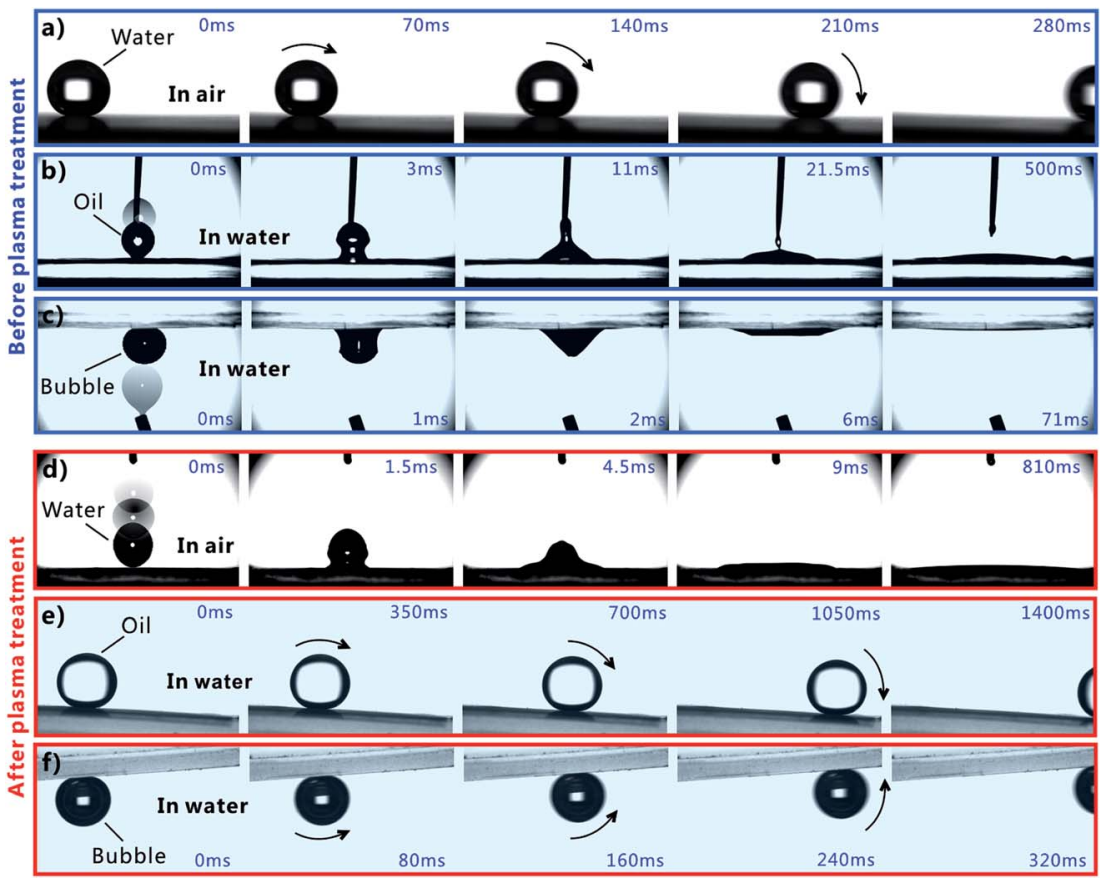

Fig. 4 Dynamic water wettability, underwater oil wettability, and underwater bubble's behavior on the femtosecond-laser-induced hierarchical rough PDMS surface. (a) Water droplet rolling on the sample surface in air. (b) Oil droplet spreading out on the sample surface in water. (c) Bubble spreading out on the sample surface in water. $(\mathrm{a}-\mathrm{c})$ Are for the PDMS surface that was just ablated by femtosecond laser. (d) Water droplet spreading out on the sample surface in air. (e) Oil droplet rolling on the sample surface in water. (f) Bubble rolling on the sample surface in water. $(d-f)$ Are for the PDMS surface that was ablated by femtosecond laser and further irradiated by oxygen plasma.

polymer. Since rough microstructures have the amplifying function on surface wettability, the formation of the hierarchical microstructure by femtosecond laser ablation finally endows the PDMS substrate with superhydrophobic property. ${ }^{1-4}$ Underwater oil wettability was measured by immersing the rough PDMS sample in water and placing an oil (1,2-dichloroethane) droplet onto the laser structured area. Once the oil droplet came in contact with the substrate, it would spread out quickly and lead to a very small oil CA of $6.5^{\circ} \pm 1.5^{\circ}$ (Fig. $3 \mathrm{c}, 4 \mathrm{~b}$ and Movie S2 in ESI†). Thus, the femtosecond laser ablated PDMS surface exhibits superoleophilicity in water medium. Similar to the underwater oil wettability, the rough PDMS surface also exhibits underwater superaerophilicity. Regarding the rough PDMS sample that was dipped into water with its structured side faced down, when a bubble was released below the sample, the bubble would rise up until it touched the rough PDMS surface. Then, the bubble was quickly and completely absorbed by the PDMS surface within $71 \mathrm{~ms}$ (Fig. $4 \mathrm{c}$ and Movie $\mathrm{S} 3$ in $\mathrm{ESI} \dagger$ ). The CA to the bubble was as low as $\sim 0^{\circ}$ (Fig. 3e). Therefore, the hierarchical rough PDMS surface after femtosecond laser ablation simultaneously exhibits superhydrophobicity, underwater superoleophilicity, and underwater superaerophilicity.

Short-time oxygen plasma irradiation is one of the most commonly used ways for activating the PDMS surface by inducing a radical silanol group $(-\mathrm{SiOH}){ }^{54,55}$ During plasma irradiation, the original $-\mathrm{CH}_{3}$ of the PDMS surface is converted into the hydrophilic group of $-\mathrm{OH} .^{54,55} \mathrm{As}$ a result, PDMS surface can be switched from hydrophobic to hydrophilic by oxygen plasma treatment. Such conversion only needs a very short exposure time, so the morphology of PDMS surface is almost unaffected. After the femtosecond laser structured PDMS surface is further irradiated by oxygen plasma for $30 \mathrm{~s}(50 \mathrm{~W})$, the sample turned to a superhydrophilic surface as shown in Fig. $3 \mathrm{~b}$. When a water droplet was dripped onto the PDMS surface in air, the droplet could fully wet the surface as the droplet rapidly spread out in every direction, finally resulting in a very small CA of $4.5^{\circ} \pm 0.5^{\circ}$ to this water droplet (Fig. $4 \mathrm{~d}$ and Movie S4 in ESI $\dagger$ ). Fig. $3 \mathrm{~h}$ is the photo of the superhydrophilic PDMS surface underwater. In this case, the mirror like reflectance disappeared. Contrary to the underwater superoleophilicity and superaerophilicity of the original laserinduced rough PDMS surface, the plasma treated rough surface repels oil droplets and air bubbles in water rather than absorbing them. In water medium, both oil droplet and bubble on the oxygen plasma treated rough PDMS surface could maintain an approximately spherical shape with the oil CA of $158^{\circ} \pm 2^{\circ}$ (Fig. 3d) and gas CA of $156^{\circ} \pm 2.5^{\circ}$ (Fig. 3f). This result indicates that the sample exhibits superoleophobicity and superaerophobicity in water. In addition, the underwater PDMS sample also shows very low adhesion to oil droplet and air bubble. The oil droplet could roll off on the $3^{\circ}$ tilted rough PDMS surface easily (Fig. 4e and Movie S5 in ESI $\dagger$ ), while the bubble could roll away as soon as the sample was tilted $3.5^{\circ}$ in water (Fig. $4 \mathrm{f}$ and Movie S6 in ESI†). Therefore, the hierarchical rough PDMS surface that is ablated by femtosecond laser and 
further irradiated by oxygen plasma simultaneously shows superhydrophilicity, underwater superoleophobicity, and underwater superaerophobicity.

It is worth mentioning that the plasma treated PDMS surface should be stored in water otherwise its original wettabilities before plasma treatment would gradually recover. The oxygen plasma treated rough PDMS surface was able to retain superhydrophilicity, underwater superoleophobicity, and underwater superaerophobicity for more than 8 months when the sample was always immersed in deionized water. By contrast, if the plasma-treated rough PDMS surface was placed in an air environment, it would restore superhydrophobicity as well as underwater superoleophilicity and superaerophilicity within $\sim 10$ hours. The main reasons for hydrophobic recovery are the reorientation of the oxygen-plasma-induced polar groups from the surface to the bulk, diffusion of the pre-existing lowmolecular-weight PDMS species from the bulk to the surface, and silanol condensation with water elimination. ${ }^{56-58}$ Surprisingly, when the oxygen-plasma-treated PDMS sample is immersed in water, the polar groups $(-\mathrm{SiOH})$ are attracted to the surface by its contact with water, that is, the high surface energy of water prevents the reorganization of the silanol groups.

The underwater oil wettability and the underwater bubble's behavior on the femtosecond laser structured PDMS surface are closely associated with the in-air water wettability of material surface. The formation mechanisms of those above-mentioned special wettabilities are depicted in Fig. 5. The femtosecond laser structured PDMS surface exhibits superhydrophobicity in air. Such superhydrophobicity is caused by the combined action of the laser-induced hierarchical microstructures and the inherent low surface free energy of PDMS. A water droplet on such surface is at the Cassie wetting state, indicating that the droplet could not wet the microstructures and is just in contact with the top peaks of the rough microstructures, as shown in Fig. 5a. ${ }^{1,53}$ An air layer is trapped between the water droplet and the surface microstructure like an air cushion underneath the droplet. Once the PDMS surface is immersed in water, the air cushion would transform into a huge trapped air layer surrounding the laser structured area irrespective of whether the structured side faces down or up (Fig. $5 \mathrm{~b}$ and e). Such an air layer gives rise to a silver mirror-like reflectance. If an underwater oil droplet is dripped onto the sample surface, the oil is able to enter into the trapped air layer and quickly spread out along the air void, through capillary action and pressure (Fig. 5c). ${ }^{47}$ This process leads to a very small CA value to this oil droplet. Moreover, the oil droplet can fully spread out as long as the rough area is large enough, revealing underwater superoleophilicity (Fig. 5d). Regarding an underwater bubble, its behavior on such rough PDMS surface is very similar with the oil wettability. When a bubble is released onto the rough PDMS surface, the air in the bubble would merge with the air in the trapped air layer once they are in contact with each other (Fig. 5f). Consequently, the air in the bubble rapidly enters into the trapped air layer under pressure. It appears that the bubble is completely absorbed by the femtosecond laser ablated PDMS surface; hence, the surface also exhibits underwater superaerophilicity (Fig. 5g).

The wettability of the femtosecond-laser-induced rough PDMS surface changes drastically after further oxygen plasma treatment. Oxygen plasma treatment can switch the rough

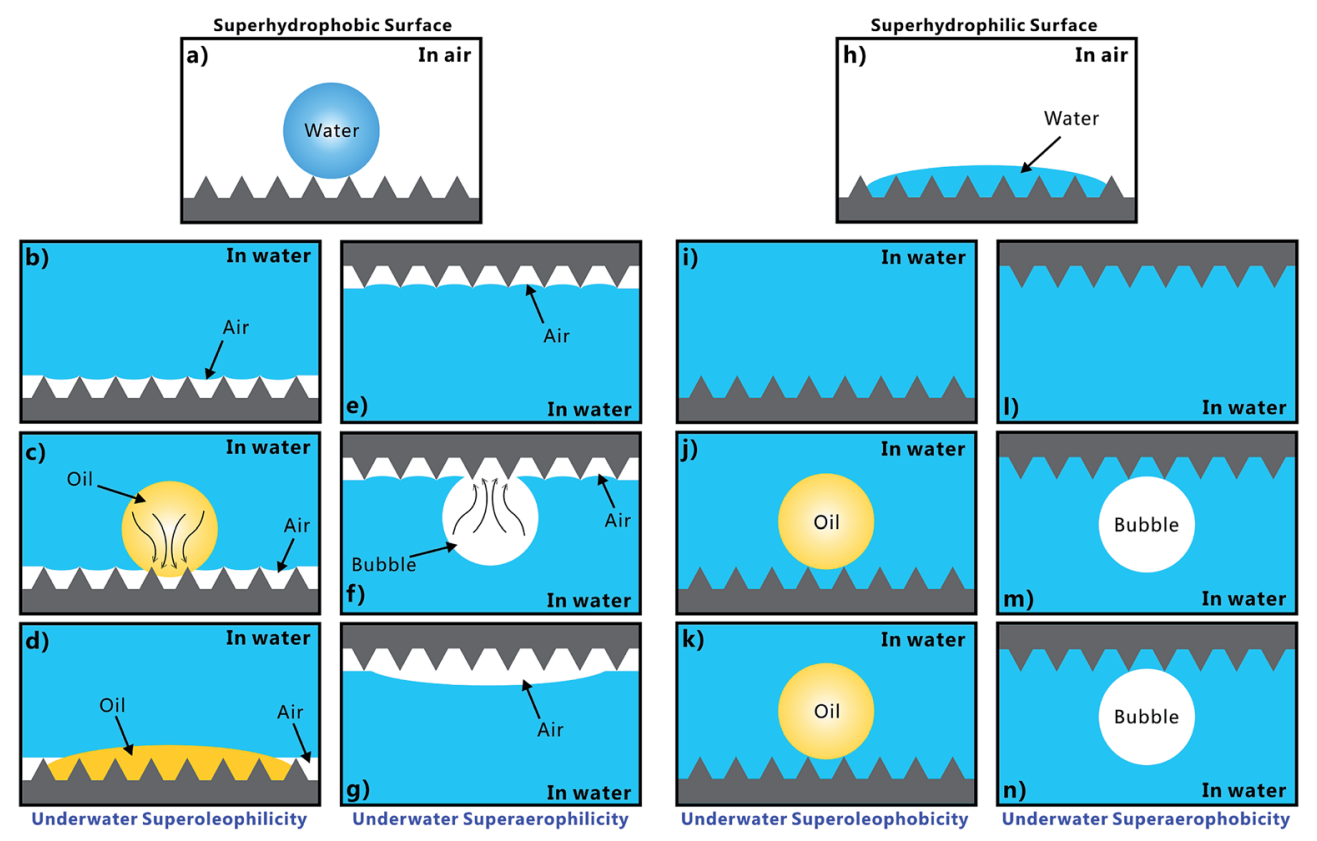

Fig. 5 Formation mechanisms of the underwater superoleophilicity and superaerophilicity of the superhydrophobic PDMS surface and the underwater superoleophobicity and superaerophobicity of the superhydrophilic PDMS surface. $(a, h)$ Water droplet on the sample surface in air. $(b, e, i, l)$ Immersion of the sample in water. (c, j) Dripping an oil droplet onto the sample surface in water. (f, $m$ ) Releasing an air bubble onto the sample surface in water. $(d, g, k, n)$ Shape changes of the oil droplet and bubble over time. $(a-g)$ Are for the PDMS surface that is just ablated by femtosecond laser. $(h-n)$ Are for the PDMS surface that is ablated by femtosecond laser and further irradiated by oxygen plasma. 
PDMS surface from superhydrophobic to superhydrophilic. Water droplet can spread out and wet such a PDMS surface in air (Fig. 5h). This wetting model belongs to the Wenzel state. ${ }^{\mathbf{1 , 5 3}}$ When the sample is dipped in water, water is able to enter into the space of the microstructure due to superhydrophilicity and fully wet the rough area (Fig. 5i and l). Furthermore, if an oil droplet and a bubble are placed on the PDMS surface, the effective contact between the oil droplet/bubble and the PDMS surface would be hindered by the water that is filled in the interspaces of the microstructures because water inherently repels oil and the bubble. Such a water layer is tightly trapped by the rough PDMS microstructure and is hard to be replaced by the oil and the bubble. The trapped water layer only allows the oil droplet and air bubble to touch the peaks of the microstructures (Fig. 5j and $\mathrm{m}$ ). Since most of the surface area of the oil droplet and the bubble is surrounded by water, the oil droplet and bubble on the oxygen plasma treated rough PDMS surface can only maintain near-spherical shapes based on the principle of minimum free-energy. Underwater superoleophobicity and superaerophobicity are exhibited by the surface. The shapes of the underwater oil droplet and bubble can be maintained on the sample surface all the time (Fig. 5k and $\mathrm{n}$ ). The oil wettability in such underwater solid/water/oil three-phase system and the bubble's behavior in such underwater solid/water/gas three-phase system can be regarded as an underwater version of Cassie state. ${ }^{1,35}$

According to the experimental and theoretical analysis discussed above, we find that the in-air superhydrophobic surface would exhibit superoleophilicity and superaerophilicity in water, while the in-air superhydrophilic surface would exhibit superoleophobicity and superaerophobicity in water. The superhydrophobicity, superoleophobicity, and superaerophobicity can endow the materials with anti-water/oil/ bubble ability. In contrast, the superhydrophilicity, superoleophilicity, and superaerophilicity can endow the materials with absorbing and capturing water/oil/bubble capacity.

Generating wettability-contrast domains on the same surface has numerous applications, such as droplets/liquid patterning. ${ }^{59-70}$ The original femtosecond laser ablated PDMS surface exhibits superhydrophobicity, underwater superoleophilicity, and underwater superaerophilicity, while the surface that is further treated by oxygen plasma exhibits
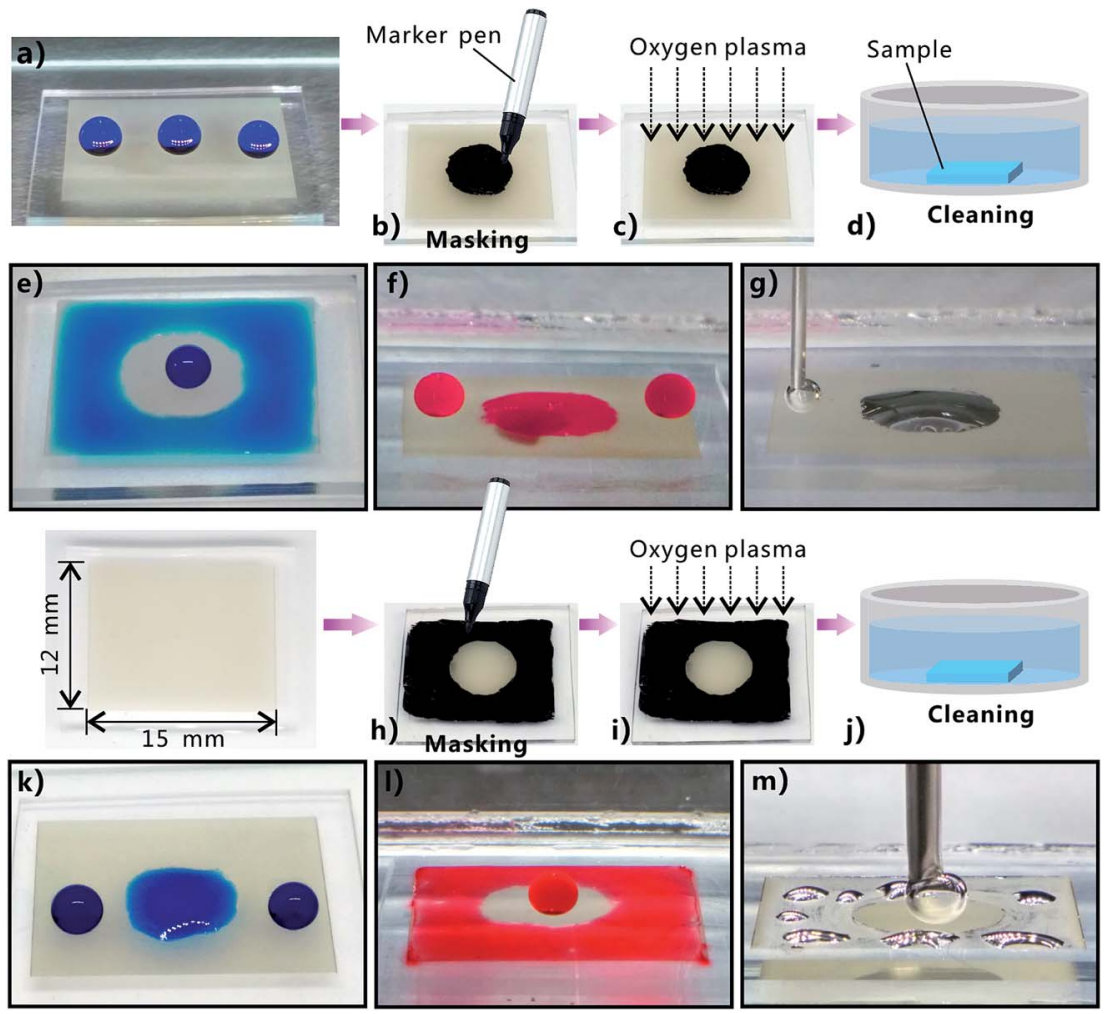

Fig. 6 Fabrication of the superhydrophobic-superhydrophilic, underwater superoleophobic-superoleophilic and underwater superaerophobic-superaerophilic hybrid patterns on the femtosecond laser ablated PDMS surface by selective oxygen plasma treatment. (a) Water droplets on the original femtosecond laser ablated PDMS surface. (b-d) Process of patterning wettability-contrast domains on the same femtosecond laser ablated PDMS surface: (b) make a mask by marker pen, (c) selective oxygen plasma treatment, (d) cleaning the sample to remove the black chemical coating. (e) Water droplets, (f) underwater oil droplets, and (g) underwater air bubbles on the resultant wettabilityhybrid PDMS surface that is composed of a non-plasma-irradiated central circular region and an oxygen-plasma-treated surrounding region. (hm) The treated process and the wettability of the resultant wettability-hybrid PDMS surface that is composed of an oxygen-plasma-treated central circular region and a non-plasma-irradiated surrounding region: $(\mathrm{h}-\mathrm{j})$ fabrication process, $(\mathrm{k})$ in-air water wettability, $(\mathrm{l})$ underwater oil wettability, $(\mathrm{m})$ underwater behavior of bubbles. For clear observation, the water was dyed with methylene blue and showed a blue color; the oil was dyed with oil red $O$ to obtain a red color. 
superhydrophilicity, underwater superoleophobicity, and underwater superaerophobicity. Such a wettability transition suggests a new method of constructuring superhydrophobicsuperhydrophilic, underwater superoleophobic-superoleophilic, and underwater superaerophobic-superaerophilic hybrid surfaces. The wettability-patterned PDMS surface can simply be obtained through femtosecond laser ablation and selectively irradiating a specific region by oxygen plasma. A simple example is shown in Fig. 6. The original femtosecond laser structured PDMS surface is superhydrophobic. Water droplets on every area of the rough sample surface can maintain spherical shape (Fig. 6a). To treat only a specific region by plasma, a mask is required. However, traditional photo-mask is not valid because the oxygen plasma has fluid property. In our experiment, a black marker pen was used to mask the area from plasma treatment (Fig. 6b). The black chemical coating covering over the PDMS surface was able to prevent the oxygen plasma from effectively coming in contact with the PDMS substrate underneath during the process of plasma irradiation (Fig. 6c). Therefore, only the non-painted region was treated by oxygen plasma. After the plasma irradiation, the sample was finally cleaned by acetone and deionized water to remove the black chemical coating (Fig. 6d). Fig. 6b-g shows the treated process and the wettability of a rectangular rough PDMS surface $(15 \mathrm{~mm}$ $\times 12 \mathrm{~mm}$ ) that is composed of a non-plasma-irradiated central circular region with a diameter of $\sim 7 \mathrm{~mm}$ and an oxygenplasma-treated surrounding region. In air, water droplet on the circular region still retained spherical shape because such an area was not activated by oxygen plasma and thus maintained its original superhydrophobicity (Fig. 6e). In contrast, when some water droplets were dripped onto the surrounding region, the droplets would spread out quickly and completely wet this region, revealing the superhydrophilicity of the surrounding region (Fig. 6e). Finally, a ring-shaped water film formed around the central circular region. When the resultant sample was immersed in water and oil (1,2-dichloroethane) droplets were dripped onto these two different regions, it was found that oil droplets fully wetted the circular region, but were repelled by the surrounding region (Fig. 6f). Therefore, the central circular region of the sample surface shows underwater superoleophilicity, while its surrounding region shows underwater superoleophobicity. The underwater behavior of air bubbles on this hybrid PDMS surface was also investigated. Similar to the underwater oil wettability, the central circular region is superaerophilic and the surrounding region is superaerophobic in water (Fig. 6g). Certainly, we can also paint the surrounding region by black marker pen and only allow the remaining central circular region to be treated by oxygen plasma as shown in Fig. $6 \mathrm{~h}-\mathrm{j}$. In this case, the central circular region exhibits superhydrophilicity (Fig. 6k), underwater superoleophobicity (Fig. 6l), and underwater superaerophobicity (Fig. 6m), while the surrounding region exhibits superhydrophobicity (Fig. 6k), underwater superoleophilicity (Fig. 61), and underwater superaerophilicity (Fig. 6m). The above results demonstrate that the superhydrophobic-superhydrophilic, underwater superoleophobic-superoleophilic and underwater superaerophobic-superaerophilic hybrid patterns were successfully achieved on the femtosecond laser ablated PDMS surface by selective oxygen plasma treatment. Since the process of using a marker pen to paint the PDMS surface appears like writing or painting, the wettability-contrast patterns can be flexibly and arbitrarily designed in a low-cost manner. This straightforward method for patterning extremely wettable-contrast hybrid surface would potentially open up a new method to manipulate water/oil droplets and air bubbles in patterning, ${ }^{59-61}$ shape controlling, ${ }^{62}$ surface microfluidics, ${ }^{63-65}$ cell micropatterns, ${ }^{66,67}$ fog-harvesting, ${ }^{68}$ microcondensation, ${ }^{69}$ and lab-on-chip devices. ${ }^{70}$

\section{Conclusions}

In summary, six different super-wettabilities were achieved on a same femtosecond laser ablated PDMS surface, including superhydrophobicity, superhydrophilicity, underwater superoleophobicity, underwater superoleophilicity, underwater superaerophobicity, and underwater superaerophilicity. Both experimental and theoretical analysis revealed that underwater oil wettability and underwater bubble's behavior on a solid surface are closely related to the in-air water wettability of the surface. The original femtosecond laser-induced rough PDMS surface exhibited superhydrophobicity in air and generally became superoleophilic and superaerophilic after immersing in water. The water wettability of the rough PDMS surface would switch to superhydrophilicity when it was further treated with oxygen plasma. Such superhydrophilic PDMS exhibited superoleophobicity and superaerophobicity in water medium. Based on such wettability transition, various pre-designed superhydrophobic-superhydrophilic, underwater superoleophobicsuperoleophilic and underwater superaerophobic-superaerophilic hybrid patterns were successfully achieved on the femtosecond laser ablated PDMS surface by selective oxygen plasma treatment. The superhydrophobic, superoleophobic, and the superaerophobic surfaces generally display strong antiwater/oil/bubble ability. On the contrary, the superhydrophilic, superoleophilic, and the superaerophilic surfaces can absorb and capture water/oil droplets and air bubbles. The preparation principle of the super-wettabilities reported in this study would have important guiding significance to researchers and engineers to effectively control water droplets, oil droplets and bubbles' behaviors on a solid surface.

\section{Conflicts of interest}

There are no conflicts to declare.

\section{Acknowledgements}

This work is supported by the National Key Research and Development Program of China under the Grant No. 2017YFB1104700, the National Science Foundation of China under the Grant No. 51335008 and 61475124, the NSAF Grant No. U1630111, China Postdoctoral Science Foundation under the Grant No. 2016M600786, the Collaborative Innovation Center of Suzhou Nano Science and Technology and the 
International Joint Research Center for Micro/Nano Manufacturing and Measurement Technologies.

\section{References}

1 J. L. Yong, F. Chen, Q. Yang, J. Huo and X. Hou, Chem. Soc. Rev., 2017, 46, 4168-4217.

2 Y. Tian, B. Su and L. Jiang, Adv. Mater., 2014, 26, 6872-6897. 3 L. Wen, Y. Tian and L. Jiang, Angew. Chem., Int. Ed., 2015, 54, 3387-3399.

4 B. Su, Y. Tian and L. Jiang, J. Am. Chem. Soc., 2016, 138, 17271748.

5 X. Yao, Y. Song and L. Jiang, Adv. Mater., 2011, 23, 719-734. 6 K. Liu, X. Yao and L. Jiang, Chem. Soc. Rev., 2010, 39, 32403255.

7 J. L. Yong, F. Chen, Q. Yang and X. Hou, Soft Matter, 2015, 11, 8897-8906.

8 S. Nishimoto and B. Bhushan, RSC Adv., 2013, 3, 671-690.

9 K. Liu and L. Jiang, Annu. Rev. Mater. Res., 2012, 42, 231-263.

10 Z. Xue, Y. Cao, N. Liu, L. Feng and L. Jiang, J. Mater. Chem. A, 2014, 2, 2445-2460.

11 L. Feng, Z. Zhang, Z. Mai, Y. Ma, B. Liu, L. Jiang and D. Zhu, Angew. Chem., Int. Ed., 2004, 43, 2012-2014.

12 J. L. Yong, F. Chen, Q. Yang, H. Bian, G. Du, C. Shan, J. Huo, Y. Fang and X. Hou, Adv. Mater. Interfaces, 2016, 3, 1500650.

13 J. L. Yong, Y. Fang, F. Chen, J. Huo, Q. Yang, H. Bian, G. Du and X. Hou, Appl. Surf. Sci., 2016, 389, 1148-1155.

14 S. Pan, A. K. Kota, J. M. Mabry and A. Tuteja, J. Am. Chem. Soc., 2013, 135, 578-581.

15 X. Gao, X. Yan, X. Yao, L. Xu, K. Zhang, J. Zhang, B. Yang and L. Jiang, Adv. Mater., 2007, 19, 2213-2217.

16 M. J. Kreder, J. Alvarenga, P. Kim and J. Aizenberg, Nat. Rev. Mater., 2016, 1, 1-15.

17 J. Lv, Y. Song, L. Jiang and J. Wang, ACS Nano, 2014, 8, 31523169.

18 A. R. Parker and C. R. Lawrence, Nature, 2001, 414, 33-34.

19 X. Liu, Y. Liang, F. Zhou and W. Liu, Soft Matter, 2012, 8, 2070-2086.

20 J. L. Yong, Q. Yang, F. Chen, D. Zhang, U. Farooq, G. Du and X. Hou, J. Mater. Chem. A, 2014, 2, 5499-5507.

21 J. L. Yong, Q. Yang, F. Chen, H. Bian, G. Du, U. Farooq and X. Hou, Adv. Mater. Interfaces, 2015, 2, 1400388.

22 J. Huo, Q. Yang, F. Chen, J. L. Yong, Y. Fang, J. Zhang, L. Liu and X. Hou, Langmuir, 2017, 33, 3659-3665.

23 A. Vitale, M. Quaglio, S. L. Marasso, A. Chiodoni, M. Cocuzza and R. Bongiovanni, Langmuir, 2013, 29, 15711-15718.

24 T. Kim and K. Y. Suh, Soft Matter, 2009, 5, 4131-4135.

25 G.-H. Kim, B.-H. Lee, H. Im, S.-B. Jeon, D. Kim, M.-L. Seol, H. Hwang and Y.-K. Choi, RSC Adv., 2016, 6, 41914-41918.

26 J. Songok, M. Tuominen, H. Teisala, J. Haapanen, J. Mäkelä, J. Kuusipalo and M. Toivakka, ACS Appl. Mater. Interfaces, 2014, 6, 20060-20066.

27 F. Shi, J. Niu, J. Liu, F. Liu, Z. Wang, X.-Q. Feng and X. Zhang, Adv. Mater., 2007, 19, 2257-2261.

28 E. P. Ivanova, J. Hasan, H. K. Webb, G. Gervinskas, S. Juodkazis, V. K. Truong, A. H. F. Wu, R. N. Lamb,
V. A. Baulin, G. S. Watson, J. A. Watson, D. E. Mainwaring and R. J. Crawforf, Nat. Commun., 2013, 4, 2838.

29 L. Shen, B. Wang, J. Wang, J. Fu, C. Picart and J. Ji, ACS Appl. Mater. Interfaces, 2012, 4, 4476-4483.

30 P. Roach, N. J. Shirtcliffe and M. I. Newton, Soft Matter, 2008, 4, 224-240.

31 J.-N. Wang, Y.-L. Zhang, Y. Liu, W. Zheng, L. P. Lee and H.-B. Sun, Nanoscale, 2015, 7, 7101-7114.

32 Y.-L. Zhang, H. Xia, E. Kim and H.-B. Sun, Soft Matter, 2012, 8, 11217-11231.

33 V. Zorba, E. Stratakis, M. Barberoglou, E. Spanakis, P. Tzanetakis, S. H. Anastasiadis and C. Fotakis, Adv. Mater., 2008, 20, 4049-4054.

34 L. Feng, S. Li, Y. Li, H. Li, L. Zhang, J. Zhai, Y. Song, B. Liu, L. Jiang and D. Zhu, Adv. Mater., 2002, 14, 1857-1860.

35 M. Liu, S. Wang, Z. Wei, Y. Song and L. Jiang, Adv. Mater., 2009, 21, 665-669.

36 T. Jiang, Z. Guo and W. Liu, J. Mater. Chem. A, 2015, 3, 18111827.

37 Z. Lu, M. Sun, T. Xu, Y. Li, W. Xu, Z. Chang, Y. Ding, X. Sun and L. Jiang, Adv. Mater., 2015, 27, 2361-2366.

38 Z. Lu, W. Zhu, X. Yu, H. Zhang, Y. Li, X. Sun, X. Wang, H. Wang, J. Wang, J. Luo, X. Lei and L. Jiang, Adv. Mater., 2014, 26, 2683-2687.

39 Z. Lu, Y. Li, X. Lei, J. Liu and X. Sun, Mater. Horiz., 2015, 2, 294-298.

40 K. Sugioka and Y. Cheng, Appl. Phys. Rev., 2014, 1, 041303. 41 D. Wu, Q.-D. Chen, L.-G. Niu, J.-N. Wang, J. Wang, R. Wang, H. Xia and H.-B. Sun, Lab Chip, 2009, 9, 2391-2394.

42 A. Y. Vorobyev and C. Guo, Laser Photonics Rev., 2013, 7, 385407.

43 J. L. Yong, F. Chen, Q. Yang, Y. Fang, J. Huo and X. Hou, Chem. Commun., 2015, 51, 9813-9816.

44 J. L. Yong, F. Chen, Q. Yang, G. Du, C. Shan, H. Bian, U. Farooq and X. Hou, J. Mater. Chem. A, 2015, 3, 9379-9384.

45 J. L. Yong, F. Chen, Q. Yang, Y. Fang, J. Huo, J. Zhang and X. Hou, Adv. Mater. Interfaces, 2017, 4, 1700552.

46 J. L. Yong, F. Chen, Q. Yang, D. Zhang, U. Farooq, G. Du and X. Hou, J. Mater. Chem. A, 2014, 2, 8790-8795.

47 J. L. Yong, F. Chen, Q. Yang, U. Farooq and X. Hou, J. Mater. Chem. A, 2015, 3, 10703-10709.

48 J. L. Yong, F. Chen, Y. Fang, J. Huo, Q. Yang, J. Zhang, H. Bian and X. Hou, ACS Appl. Mater. Interfaces, 2017, 9, 39863-39871.

49 J. L. Yong, F. Chen, Q. Yang, D. Zhang, H. Bian, G. Du, J. Si, X. Meng and X. Hou, Langmuir, 2013, 29, 3274-3279.

50 J. L. Yong, F. Chen, Q. Yang, D. Zhang, G. Du, J. Si, F. Yun and X. Hou, J. Phys. Chem. C, 2013, 117, 24907-24912.

51 I. A. Larmour, S. E. J. Bell and G. C. Saunders, Angew. Chem., 2007, 119, 1740-1742.

52 Y. Zhao, Y. Tang, X. Wang and T. Lin, Appl. Surf. Sci., 2010, 256, 6736-6742.

53 S. Wang and L. Jiang, Definition of superhydrophobic states, Adv. Mater., 2007, 19, 3423-3424.

54 D. Wu, S. Wu, Q.-D. Chen, S. Zhao, H. Zhang, J. Jiao, J. A. Piersol, J.-N. Wang, H.-B. Sun and L. Jiang, Lab Chip, 2011, 11, 3873-3879. 
55 Y. Cai, L. Lin, Z. Xue, M. Liu, S. Wang and L. Jiang, Adv. Funct. Mater., 2014, 24, 809-816.

56 M. Morra, E. Occhiello, R. Marola, F. Garbassi, P. Humphrey and D. Johnson, J. Colloid Interface Sci., 1990, 137, 11-24.

57 H. Hillborg, J. F. Ankner, U. W. Gedde, G. D. Smith, H. K. Yasuda and K. Wikström, Polymer, 2000, 41, 68516863.

58 S. H. Tan, N.-T. Nguyen, Y. C. Chua and T. G. Kang, Biomicrofluidics, 2010, 4, 032204.

59 S. P. Kobaku, A. K. Kota, D. H. Lee, J. M. Mabry and A. Tuteja, Angew. Chem., Int. Ed., 2012, 51, 10109-10113.

60 W. Feng, L. Li, E. Ueda, J. Li, S. Heißler, A. Welle, O. Trapp and P. A. Levkin, Adv. Mater. Interfaces, 2014, 1, 1400269.

61 A. N. Efremov, M. Grunze and P. A. Levkin, Adv. Mater. Interfaces, 2014, 1, 1300075.

62 V. Jokinen, L. Sainiemi and S. Franssila, Adv. Mater., 2008, 20, 3453-3456.
63 D. Zahner, J. Abagat, F. Svec, J. M. J. Fréchet and P. A. Levkin, Adv. Mater., 2011, 23, 3030-3034.

64 H. S. Khoo and F.-G. Tseng, Appl. Phys. Lett., 2009, 95, 063108.

65 A. Ghosh, R. Ganguly, T. M. Schutzius and C. M. Megaridis, Lab Chip, 2014, 14, 1538-1550.

66 G. Piret, E. Galopin, Y. Coffinier, R. Boukherroub, D. Legrand and C. Slomianny, Soft Matter, 2011, 7, 86428649.

67 W. Shi, T. Xu, L.-P. Xu, Y. Chen, Y. Wen, X. Zhang and S. Wang, Nanoscale, 2016, 8, 18612-18615.

68 Y. Wang, L. Zhang, J. Wu, M. N. Hedhili and P. Wang, J. Mater. Chem. A, 2015, 3, 18963-18969.

69 R. P. Garrod, L. G. Garris, W. C. Schofield, J. McGettrick, L. J. Ward, D. O. H. Teare and J. P. S. Badyal, Langmuir, 2007, 23, 689-693.

70 E. Ueda and P. A. Levkin, Adv. Mater., 2013, 25, 1234-1247. 